\title{
Application Research of Ontology-enabled Process FMEA Knowledge Management Method
}

\author{
Zhao Xiuxu \\ School of Mechanical \& Electrical Engineering \\ Wuhan University of Technology \\ Wuhan, China \\ zhaoxiuxu@whut.edu.cn \\ Zhu Yuming \\ School of Mechanical \& Electrical Engineering \\ Wuhan University of Technology \\ Wuhan, China \\ zym522006@163.com
}

\begin{abstract}
Failure Mode and Effect Analysis (FMEA) is an important method to ensure the effectiveness of manufacturing process, which can prevent the happening of various potential failure. If the knowledge of FMEA can be accumulated and utilized reasonably, the quality management in manufacturing process will get decision support timely, and the efficiency of product quality problem analysis also can be improved. But, it is impossible to integrate the dispersive FMEA knowledge in the manufacturing process because there is a lack of unified management criterion of FMEA knowledge. In order to satisfy the requirement to share, reuse, and maintain FMEA knowledge, the representation method of FMEA knowledge based on ontology is put forward in this study. The structure of FMEA knowledge ontology can be described via visual modeling tool- Unified Modeling Language. In this study, the FMEA repository has been built and the acquisition, storage and searching of FMEA knowledge has been researched to satisfy the need of sharing and reusing of the FMEA knowledge in the manufacturing process.
\end{abstract}

Index Terms-Failure mode and effect analysis, ontology, FMEA knowledge structure model, FMEA repository

\section{INTRODUCTION}

FMEA(Failure Mode and Effect Analysis), as an effective method and tool for procedure analyzing and risk assessing, is capable of offering critical assistance to analysis and improvement in manufacturing process[1]. As for a myriad of corporations, successfully implementing FMEA is a crucial way to ensure the effectivity in manufacturing and improving the quality of products. A variety of information will be collected based on the process predicting and analyzing of the possible failure by technical panel. That information is constructive to manufacturing process analysis and cumulating knowledge for improvements. Besides, collecting and organizing of knowledge obtained from FMEA will be helpful to reuse and share the FMEA knowledge, which are basic resources for quality administration. During manufacturing process, apposite use of FMEA knowledge is a key promise of continuous improvement in manufacturing process .

Currently, many corporations are not able to utilize FMEA effectively for feasibility and improvement in manufacturing processes, which is mainly due to a lack of applicable and effective management systems. Without a unifying standard for FMEA knowledge structure, dispersive FMEA knowledge can't be collected during manufacturing process which would satisfy the requirements to share, reuse and maintain FMEA knowledge in producing process. Thus it seriously affects implementation effect that FMEA would boost in manufacturing corporations.

Fortunately, this bottleneck has attracted some scholars' attention and some research has been done. Generally, these studies can be divided into two categories:

One is computer-aided FMEA process software, such as Relex software, and FMEA software based on knowledge modeling[2] (P.C. Teoh, 2004). They may somehow helpful, but the FMEA knowledge is hardly reusable due to the FMEA-related information is acquired in natural language, lack of uniform, consistent and semantic expression of FMEA knowledge.

The other is ontology-based FMEA knowledge management studies $[3,4,5,6,7]$. Burton H. Lee(2001) firstly presents an approach bringing ontology and FMEA explicitly together. But his approach suffers from missing explicit definition of ontology and his understanding of ontology is a conceptual model with no rules and inference. Lars Dittmann et al(2005) focuses on combining knowledge management and quality management systems for reusing the existing FMEA knowledge easily. Ahmed Laaroussi et al(2007) develop an innovative software specifically developed to perform more easily FMEA on building components 
based on structured knowledge base and an inference rule engine by ontology. V. Ebrahimipour(2009) builds an FMEA Ontology and evaluates its retrieval about relevant information using JTP inference through cases. Martin Molhanec et al (2010) propose a method of improvement of a FMEA procedure in the field of reflow lead free soldering by the use of an ontology paradigm, but he is still under research.

They all have done a lot in reusing FMEA knowledge, but just as Dittmann said "A commonsense ontology is needed that provides parts of standardized components and a functional taxonomy3." That is to say FMEA knowledge sharing and interoperation may still have some difficulty without a standard and common-understanding FMEA ontology Model.

Thence, this paper proposes an ontology-based express method of FMEA knowledge which aims to extract and convert of existing FMEA knowledge effectively; and then develops a systematic, structured and ontology-based FMEA knowledge management system. Through this method, intelligent inquiry and reasoning of FMEA knowledge can be achieved timely, and effectively decision-making knowledge to support and promote the handling of quality issues in the manufacturing process can be acquired.

The thesis is divided into three main parts, first part describes the ontology-based representation method of FMEA knowledge, the second part illustrates how to build FMEA repository and the third part is about how to execute the acquisition, storage and retrieval FMEA knowledge in the manufacturing process.

\section{PERFORMANCE OF KNOWLEDGE IN FMEA IMPLEMENT OF MANUFACTURING PROCESS}

\section{A. How FMEA Works}

Failure Mode and Effects Analysis is one of many techniques used to evaluate and analyze manufacturing process risk[8]. It should be conducted mostly in early phases of manufacturing process. The Figure 1 shows the procedure of FMEA.

Firstly, we analyse the function hierarchy of the product, the system is decomposed into subsystem, subsystem into components, components into parts; then determination of the failure modes, the cause and effect of the failure modes based on the function hierarchy of the product. Experience of crossfunctional team, data from validation test failures and field failures of similar products are structured using a check-list to quantify the value for failure Probability (O), impact (or severity) on the customer(S), and the capacity of the testing mechanisms and simulations to detect a possible failure (D). The FMEA history data of the similar products is extremely helpful in getting the possible failure modes. After that we calculate the value of $\mathrm{RPN}=\mathrm{S} \times \mathrm{O} \times \mathrm{D}$.

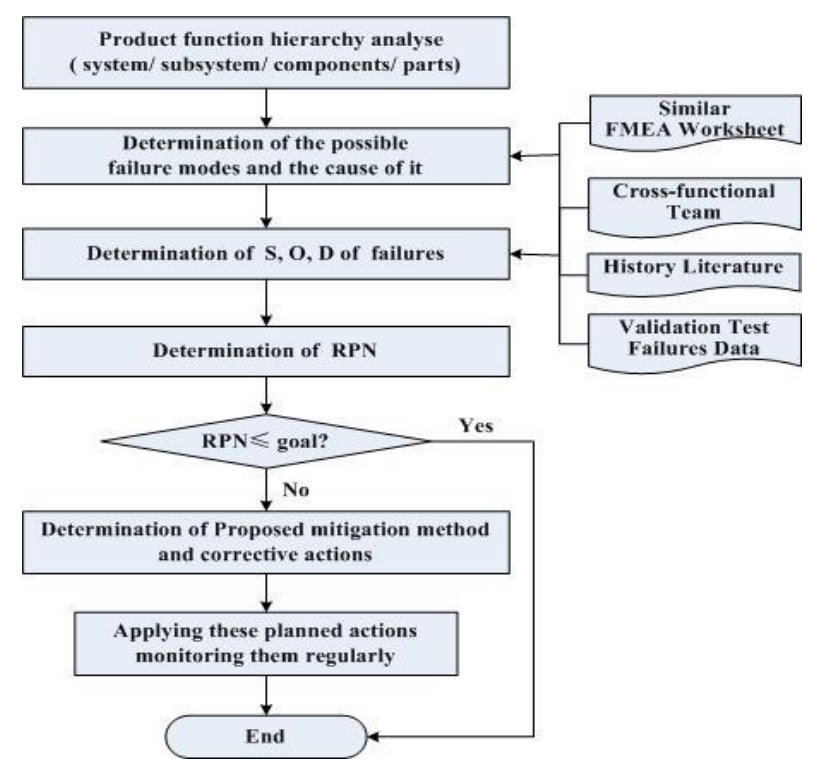

Figure 1. The procedure of FMEA in manufacturing process

If RPN is under the value which can be accepted, then the procedure can be ended, and fill the FMEA standard check-lists. If RPN is higher than acceptable value, then proposed mitigation method, corrective actions or compensating provisions must be taken, most importantly, these actions should be running as planned and be monitored regularly. If these have been done, calculate the RPN again to ensure whether it lower or higher than acceptable value. If lower, the procedure ends, if not, then continues until it is acceptable. FMEA is a living document[8], it should:

- Be initiated before or at the feasibility stage;

- Be initiated prior to tooling for production;

- Take into account all manufacturing operations from individual components to assemblies, and Include all processes within the plant that can impact the manufacturing and assembly operations, such as shipping, receiving, transporting of material, storage, conveyors or labeling;

- Be updated with process going on accordingly.

It is the history FMEA knowledge and experts' knowledge that greatly matters when conducting FMEA. Without them, FMEA can hardly perform its function. If they can be reused and shared, implementing FMEA will become much easier and more efficient.

\section{B. The Analysis of Characteristics of FMEA Knowledge in Manufacturing Process}

Hence, the study begins with analysis of process FMEA (PFMEA) knowledge characteristics, it can be summarized as the follows:

- Extensity, FMEA knowledge involves a wide range of process knowledge, both internal and external business process knowledge;

- Implicity, a great part of FMEA knowledge is the experience and skills in the experts' mind, it is difficult to express clearly by using natural language; 
- Diversity, FMEA knowledge exists in the various forms, such as words, symbols, graphics and tables, equations and so on;

- Complexity, as for manufacturing process, FMEA knowledge is the multi-disciplinary knowledge of mechanical, material, manufacturing process and quality management knowledge and is also closely related with enterprise resources.

Therefore, considering the characteristics of FMEA knowledge in manufacturing process, seeking a knowledge representation method to provide an unified consensus FMEA knowledge for the sharing and reuse of FMEA knowledge is a critical problem that should be solved in the FMEA knowledge management. Most importantly, the representation method of FMEA knowledge is the theoretical basis of successfully constructing FMEA knowledge system.

\section{CONSTRUCTION METHOD OF FMEA DOMAIN ONTOLOGY REPOSITORY}

\section{A. Ontology-based Representation Method ofFMEA Knowledge}

Ontology, the concept of which firstly originated in the field of philosophy, is a systematic interpretation or explanation of the objective existence, emphasizing the abstract nature of the objective reality[9]. By using Ontology in knowledge management field, the isolated, fragmented and unrelated knowledge can be transformed into interrelated, systematic and structured knowledge, therefore, the knowledge can be used and searched efficiently. Because ontology can describe the concept of hierarchy well and support the logical reasoning somehow, it has been widely used in knowledge-sharing-orented applications field[10].

In the field of manufacturing industry informationization, Ontology can make the relations of concepts, the relations between concepts and objects, and the relations between objects and objects all clearly in problem domain, thus ,reducing the misunderstanding between concepts and logical relations in the problem-sloving process, and knowledge can be widely shared and reused in this way. As a result, Ontology is considered as a solution to integrate multi-source and heterogeneous data, information and knowledge layer in the manufacturing information system[11,12]. Ontology can not only provide a feasible way to express FMEA repository knowledge in the manufacturing process, but also give a normalized description to its concepts, terms, and the relationship between them, help to establish a complete structured system in the expression of domain knowledge.

As Ontology has outstanding advantages in knowledge sharing and reuse, this paper presents ontology-based FMEA knowledge representation method in the manufacturing process. Through normalized description to the knowledge's concepts , terms, and the relationship between them to establish the architecture of FMEA domain knowledge. FMEA domain ontology provides common understanding of FMEA domain knowledge and becomes the semantic basis of mutual understanding between machines and man-machines by giving accurate description to the relationship between concepts; contributes to reach a consensus on understanding of FMEA domain concepts and provides a systematic and structured approach to develop FMEA ontology repository, and achieves an effective integration way of FMEA knowledge management in the manufacturing process, which can support the continuous improvement in the manufacturing process.

Applying ontology to the repository, terms or concepts are used to express knowledge, and the intrinsic relations between these knowledge are revealed at the same time. The knowledge in the Ontology-based repository system is not only well classified as class-subclass in the vertical direction, but also through the ontology's association to execute organization, association and reasoning among knowledge to meet the users' different needs for knowledge[13]. The construction of FMEA ontology repository mainly included representation, use and acquisition of FMEA knowledge to meet the users' needs in manufacturing process.

\section{B. UML-based Visual Expression of FMEA Knowledge Structure}

In order to create FMEA do main ontology repository in a systematic and structured way, this paper uses UML (Uniform Modeling Language) ,a class diagram, to express the structural model of FMEA domain knowledge. Uniform Modeling Language (UML) is a universal visual modeling language used to describe software, visually process, construct and create software systems documentation[15]. In this study, the UML visual modeling language is used to express specific FMEA ontology knowledge, to describe the hierarchy of the concept of FMEA knowledge and to illustrate the relations between concepts and objects clearly. Table I shows three main UML language notations.

TABLE I.

BASIC UML LANGUAGE NOTATIONS

\begin{tabular}{|l|l|}
\hline $\begin{array}{c}\text { The language symbol of } \\
\text { UML }\end{array}$ & The semantic specification of symbol \\
\hline \hline & $\begin{array}{l}\text { Indicate the concepts and object in } \\
\text { system; }\end{array}$ \\
\hline & $\begin{array}{l}\text { Indicate the relations bet ween } \\
\text { concepts, concept and object; }\end{array}$ \\
\hline & $\begin{array}{l}\text { Indicate the inclusion relation } \\
\text { between up-level concept and down- } \\
\text { level concept, such as "part of "; }\end{array}$ \\
\hline
\end{tabular}

FMEA knowledge in the manufacturing system can be regarded as the composition of a set of failure modes of components, manufacturing processes, process measurements and processing procedures. A particular 
component has a series of manufacturing processes, and each process has a certain size requirements, thus, various failure modes may occur in this process. Fig. 2 shows the hierarchical relation graph of a specific process.

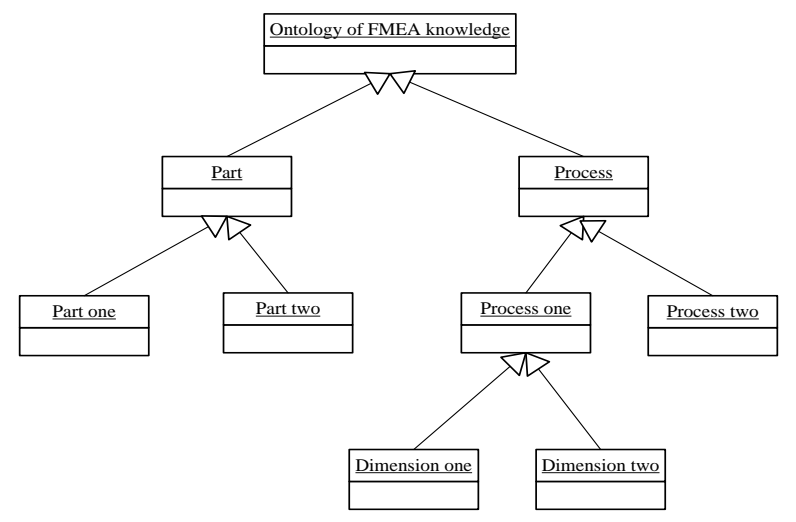

Figure 2. Hierarchical model of FMEA repositoryd on UML

In order to achieve effective management of FMEA knowledge in manufacturing system, the relations between the FMEA ontology domains must be clearly expressed. Fig.3 shows the interrelations of components, process, process size and failure modes.

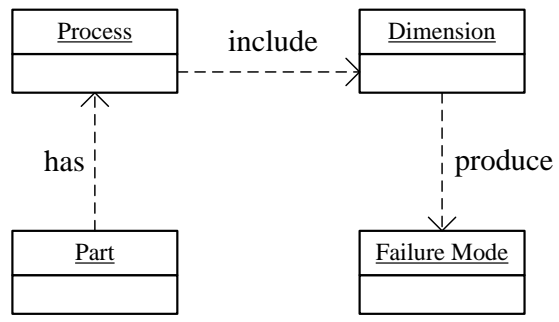

Figure 3. Relations between area ontology of FMEA

- The relationship of manufacturing process: as for a specific process, all processes have the obvious orders, which is shown in Fig.4.

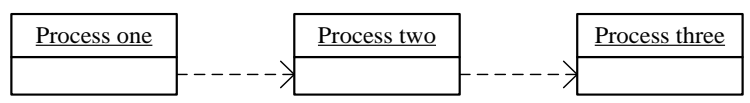

Figure 4. Relations between processes

- The relation graph of failure modes: as for a specific failure mode, it contains a series of attributes and the specific attribute values. Fig.5 shows their interrelations.

In a conclusion, the hierarchy relations between concepts, as well as the relations between concepts and objects of FMEA knowledge ontology can be expressed via visual graphs by using basic UML language notations. On this basis, the vertical and horizontal inter-association relations of FMEA knowledge can be further clarified, which contributes to create FMEA knowledge conceptual model, clarify the relations of FMEA knowledge and make the further application of FMEA knowledge ontology possible.

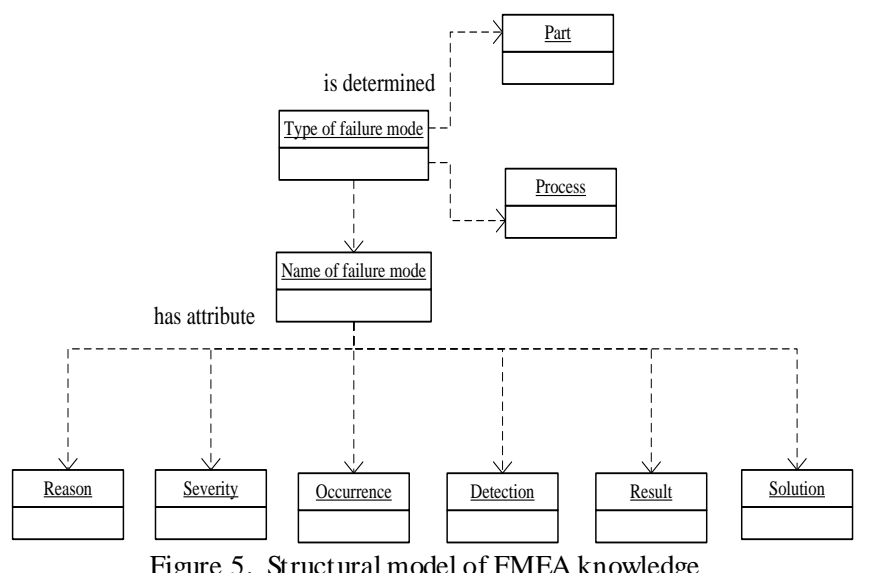

\section{Development of Ontology-based FMEA knowledge base}

FMEA knowledge exists in various forms, mainly includes explicit knowledge, which usually exists in text format(such as Word, Excel) and DB forms, and tacit knowledge, which is mainly stored in the experts' minds who participate in the FMEA process and is difficult to extract. In the implementation of the FMEA process, such tacit knowledge can be effectively extracted by using "brainstorming". Moreover, by creating FMEA domain ontology, further sharing of FMEA knowledge can be achieved[14]. The creation of FMEA domain ontology will reduce the divergences generated by the concepts and terms and reach consensus understanding according to the domain concepts.

Protégé, an extensible, cross-platform and open source development environment to create and edit ontology and repository, was developed by Stanford University in the United States. It can be used to define class and class hierarchy, attribute relations and attribute-value restriction and class-attribute relations[16]. The core modules of Protégé are mainly used to manage repository system, including knowledge representation and the establishment of knowledge hierarchy, acquisition and management.

FMEA knowledge ontology was built by Protégé as following steps:

- Description of the concept hierarchy: using the upper and lower relationship in Protégé to reflect the relationship of class and subclass in FMEA knowledge, to hierarchize the knowledge of FMEA; For example, there is "subclass_of" relationship between product and component, component and part, part and its function, function and its failure mode;

- Adding property: using equivalent and correlation relationship in Protégé to display the multiattribute relationships of the concept layers and internal structure of concepts, such as Object Property, Data Property and Annotation Property; 
Such as a failure mode has "severity, reason, occurrence, detection, result and solution" property;

- Adding instance: the instance corresponding to one or more ontology concepts, have specific attributes and attribute values;

- Adding axiom: added some constraints among the concepts, instances and attributes of FMEA knowledge; such as inverse relation, function relation, transfer relation, symmetric relation, inheritance relation, brotherhood relation, exclusive relation, partial order relation, etc. For example, inverse relation exsits between "has_failuremode" and "is_failuremode", which describes the relationship between function and its failure mode.

Figure 6 shows the concept hierarchy of an application example.

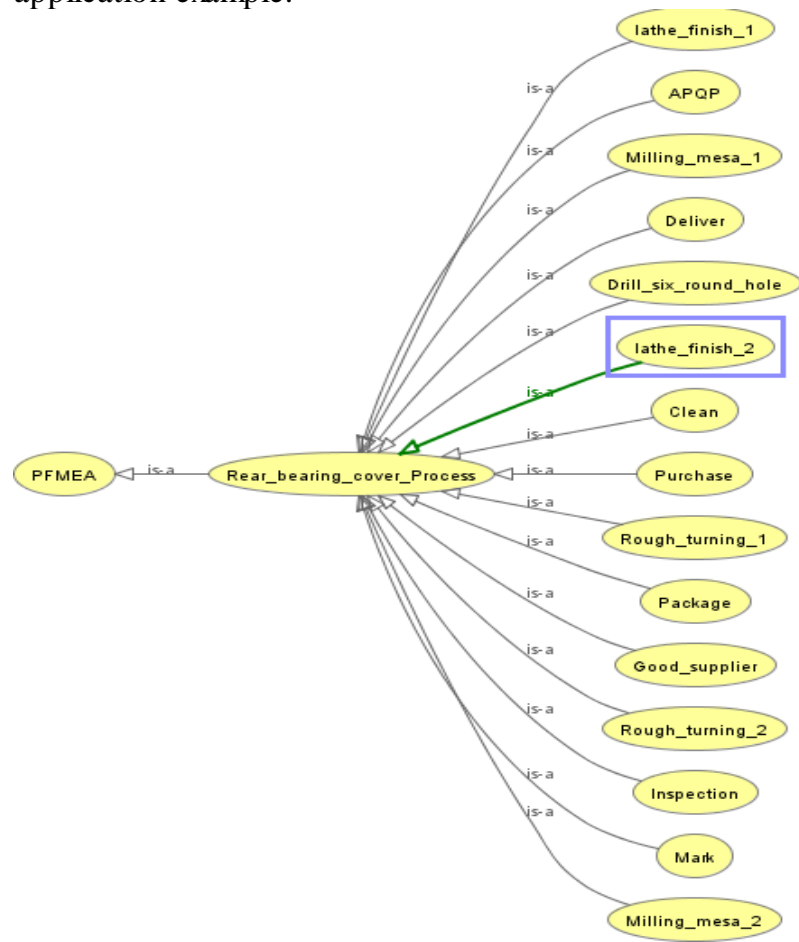

Figure 6. The hierarchy of PFMEA ontology

The first hierarchy is PFMEA, and then process, last hierarchy is detailed process. Rear bear cover process includes APQP, choosing supplier, purchase, inspection, rough turning 1 and 2, lathe finish 1 and 2, Drill six round hole, milling mesa 1 and 2, mark, clean, package and deliver.

The sequence of every process is strictly provisioned, and they interact. "is_a" shows the relation between subclass and class. "APQP, lathe_finish_2, package and ect." is the subclass of "Rear_bearing_cover_Process", which is the subclass of "PFMEA". Every detailed process has its failure mode, here we call it instance of the detailed process; every failure mode (instance) has its data property such as "S,O,D, RPN, Reason, Result and Solution", and also one failure mode may be caused by more than one reason. Just as figure 7 shows, "lathe_finish_2" has six failure modes, the failure mode "diameter of bore is under the lower tole rance, that is to say size one $<125.99 \mathrm{~mm}$ or size two $<113.00 \mathrm{~mm}$ ", and the failure mode "diameter of bore is under the lower tolerance" is caused by three reasons, there are "worker's non-standard operation; NC program fault; tool compensation inadequacy". Each failure mode will be measured by "S,O,D, RPN, Reason, Result and Solution", which is the main parameter of FMEA. If all these are done, then the PFMEA worksheet is perfectly mapped to the PFMEA ontology in protégé.

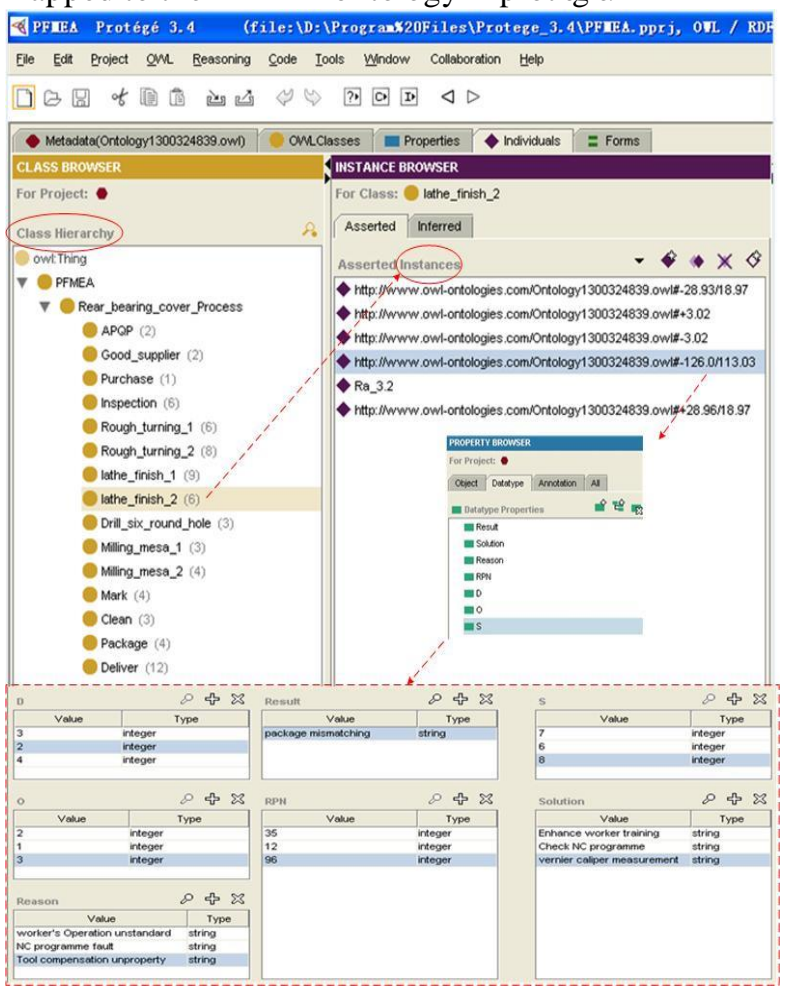

Figure 7. PFMEA ontology building by protégé 3.4

After building a PFMEA ontology, through interaction with the user, Protégé system provides an effective way to realize the FMEA knowledge intelligent query and reasoning by multi-level semantic reasoning. Using Protégé in FMEA knowledge management, can not only reflect the relations between the horizontal and vertical network of FMEA ontology domain knowledge, but also can provide the Racer inference engine to aid the detecting of the FMEA ontology establishment errors, and the intelligent reasoning of FMEA knowledge.

\section{APPLICATIONS OF FMEA ONT OLOGY REPOSITORY IN THE MANUFACT URING PROCESS}

Based on these studies, FMEA knowledge ontology was integrated into the FMEA knowledge management system in manufacturing process, by using the ontology application development tools (HP Labs) Jena 2 , and combining with the mainstream technology of development Web JSP + JavaBean, the system was designed into a Java-based B/S structure, and Eclipse + JDK was used to develop system's interface.

The structure of the application system is shown in 
Fig.8:

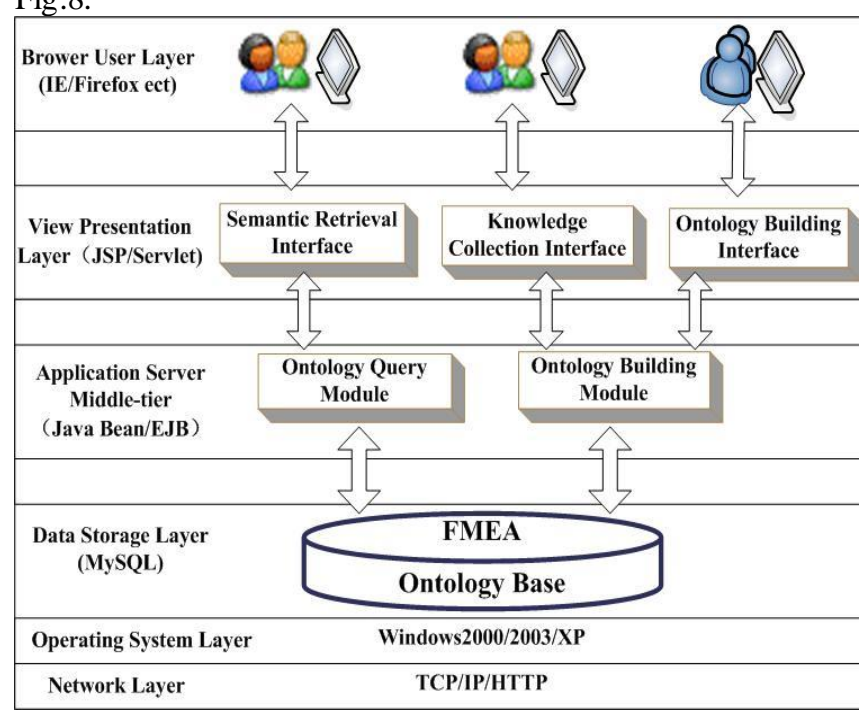

Figure 8 . The structure of the application system

The ontology query module and building module are all Jena-based modules. Jena 2 is a powerful tool to develop ontology:

- It includes Jena RDF/OWL package which can read, inquire, create and modify RDF /OWL model; what's more;

- It provides ARQ inquiry engine, provides SPARQL and RDQL query language to support ontology model querying. In addition, the inquiry engine has a fairly well interface with relational database(MySQL), which makes inquiry ontology storage in the relational database more efficiency;

- Jena 2 can achieve simple rule-based reasoning, it allows other inference engines embedded in its reasoning mechanism, to achieving associated reasoning; enabling more accurate and sensitive reasoning, digging more hidden information.

The performance of FMEA knowledge reusing and sharing are largely determined by the accuracy and comprehensiveness of more relative retrieval information. This study achieved SPARQL and RDQL query by means of ARQ query engine provided by Jena 2. In addition, through simple reasoning by means of the internal or external inference engine provided by Jena 2 and Racer inference engine which is embedded in Jena 2, more tacit and associated information can be acquired.

For example, a user can log in the system via IE browser under the authorization, inputs the key words of "manufacturing process system" in the query engine, through the reasoning system, search results was obtained: top layer "process and the size" and lower layer "various types of failure modes" of "manufacturing process", as well as a variety of attributes such as "severity", " occurrence" and so on. Moreover, the user can get more detailed information through the corresponding operations according to specific needs. Through the application system, the query precision of FMEA knowledge was greatly improved in the manufacturing process, and the rate of
FMEA information sharing and reuse can also be enhanced.

Take the rear bearing cover which is mentioned in Section III Part C for example, the company we cooperate provides parts to different automobile companies; the rear bearing cover almost varies with the automobile model, so there are more than one type rear bearing covers; implement of PFMEA it seems is somehow a repeated work, because majority of the information can be reused, actually the company almost makes a fresh start every time, making PFMEA process time-costing and boring, even discouraging the FMEA cross-functional team members; they are anxious about why they cannot reuse and share FMEA knowledge more efficient before. After this project, this problem will be handled.

\section{CONCLUSION}

In order to meet the practical need to acquire FMEA knowledge in manufacturing process, this study gives an expressive method for FMEA knowledge based on ontology, and constructs FMEA repository based on ontology. On this basis, acquisition, storage and retrieval of FMEA knowledge during manufacturing process are achieved and requirements are satisfied for the manufacturing process quality continuous improvement via further application of FMEA knowledge ontology. Ultimately, combining with the industrial practice, this study offers an effective approach for the internal of corporations, corporation transactions to share and reuse FMEA knowledge to support the decision-making in the manufacturing process.

\section{REFERENCES}

[1] Li-Feng Xi and Gang Xu, "The Application of FMEA in Process Management," J.Industrial Engineering and management.vol.1,pp 37-39, 2002.

[2] P.C. Teoh*, Keith Case, "Failure Modes and Effects Analysis through Knowledge Modeling"J.Materials Processing Technology, 2004,pp.153-154 : 253-260.

[3] Lee BH, “Using FMEA models and ontologies to build diagnostic models", J.AI Edam-Artificial Intelligence For Engineering Design Analysis And Manufacturing,vol.15,pp 281-293,2001.

[4] Lars Dittmann, Tim Rademacher, Stephan Zelewski, "Combining Knowledge Management and Quality Management Systems”, J.IEEE press,pp.11-19,2005.

[5] Ahmed Laaroussi,Bruno Fiès, Rémi Vankeisbelckt, Julien Hans, "Ontology-aided FMEA for Construction Products”,J.IEEE press,pp.189-194,2007.

[6] V. Ebrahimipour *, K. Rezaie, S. Shokravi, "An Ontology Approach to Support FMEA Studies", J.Expert Systems with Applications, vol37,pp.671677,2010

[7] Martin Molhanec, Pavel Mach, David Asamoah Bamfo Mensah, "The Ontology based FMEA of Lead Free Soldering Process", $33^{\text {rd }}$ Int. Spring Seminar on Electronics Technology, IEEE Press,pp.360-364,2010. 
[8] Potential Failure Mode and Efffects Analysis (FMEA) ReferenceManual $4^{\text {th }}$ Edition,Chry sler LLC, Ford Motor Company, General Motors Corp. (C2008

[9] STUDER R,BENJAMINS $\mathrm{V} R$ and FENSEL D, "Knowledge engineering : principles and methods," J.Data and Knowledge Engineering.vol.25.pp161-197, 1998.

[10] Zhi-Hong Dun and Shi-Wei Tang, "Ontology research summary," J. Acta of Peking university.Natural science version.vol.38.pp 730-737, 2002. (in Chinese)

[11] Qing-Ni Yuan, Qing-Sheng, Xie; Ming-Heng Xu and Shao-Bo Li, "Research on Manufacturing Resources Ontology Model Based on Semantic," Jurnal of Wuhan university of technology, Vol.31 No.10, pp 121-125, 2009. (in Chinese).

[12] Yi-Sheng Dai and Xiu-Ling Hei, "Ontology modeling of production management process in manufacturing enterprises," Journal of Harbin Engineering University, Vol.29 No.8, pp877-881, 2008. (in Chinese).

[13] Ling-Zhi Li, "Construction of Ontology-based Knowledge System," J. Information Intelligence Development and Economy.vol.17 No.36,.pp 102-104, 2007.

[14] James Rumbaugh, Ivar Jacobson and Grady Booch, "The Unified Modeling Language Reference Manual,'Peking: Machinery Industry Press, January 2001.

[15] Xiu-Xu Zhao and Xiao-Li Bai, "Research on the Construction Methods of Ontology-based FMEA Knowledge Base" Jurnal of Wuhan university of technology (Information \& Management Engineering), Vol. 31 No. 1, pp87-90, 2009. (in Chinese).

[16]Xian-Yi Chen, Yi-Song Liu and Li Yan, “Agent-oriented knowledge engineering,"Peking: Science press, 2008.

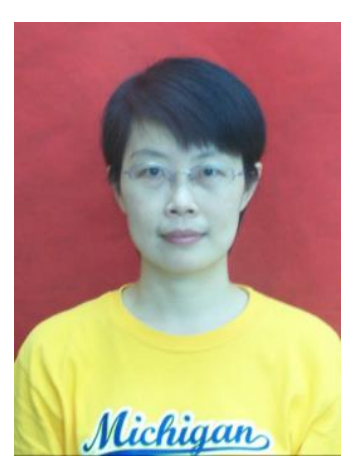

Thao Xiuxu is born in Kuidun Xingjiang China, born in September 25,1968. Between the year 1993 and 1996, gets Master degree in mechanical engineering in Wuhan Auto Industry University which is in Wuhan China. Between the year 2000 and 2004, gets $\mathrm{PhD}$ degree in mechanical engineering in Wuhan University of Technology which is in Wuhan China. Between the year 2005 and 2006, she was a visiting scholar of Wu Manufacturing Research Center which is in University of Michigan.

She is now an associate professor in Wuhan University of Technology, and work in Industrial Engineering Department in the school of Mechanical \& Electrical Engineering. Her previous publications include [1] The Application Research of Intelligent Quality Control Based on Failure Mode and Effects Analysis Knowledge Management [Book Chapter]. Applications and Experiences of Quality Control, ISBN 978953-307-236-4, Edited by: Prof. Ogny an Ivanov, Feb. 2011. [2] WC-Co Tool failure Analysis and the grinding effect study [J], Advanced Materials Research Vols.139-141 2010:269-273, Trans Tech Publications, Switzerland. [3] WC-Co tool failure mechanism in titanium alloys machining [J]. ttp TRANS TECH PUBLICATIONS, Key Engineering Materials 2008, V 368-372 PART 2:1137-1139 etc. Her current and previous research interests include industrial operation management, manufacturing process quality control, and enterprise informatization.

Dr. Zhao is the member of Chinese Mechanical Engineering Society, and got the third prize for Progress in Science and Technology of Wuhan in 2006.

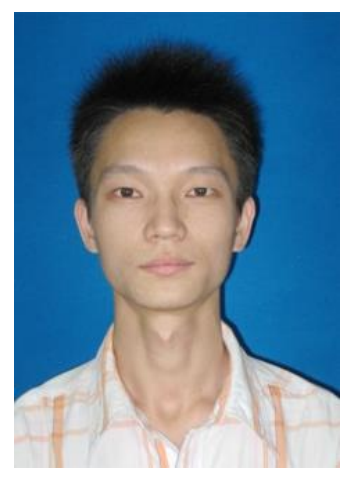

Zhu Yuming is born in GanZhou Jiangxi China, born in November 10,1986 . Between the year 2005 and 2009, gets bachelor's degree in Industrial Engineering in Wuhan University of Technology which is in Wuhan China.

$\mathrm{He}$ is now a graduate student researching in Production Operation \& Enterprise Informatization in Wuhan University of Technology

How to cite this paper: Zhao Xiuxu,Zhu Yuming,"Application Research of Ontology-enabled Process FMEA Knowledge Management Method", International Journal of Intelligent Systems and Applications(IJISA), vol.4, no.3, pp.34-40, 2012. DOI: 10.5815/ijisa.2012.03.05 